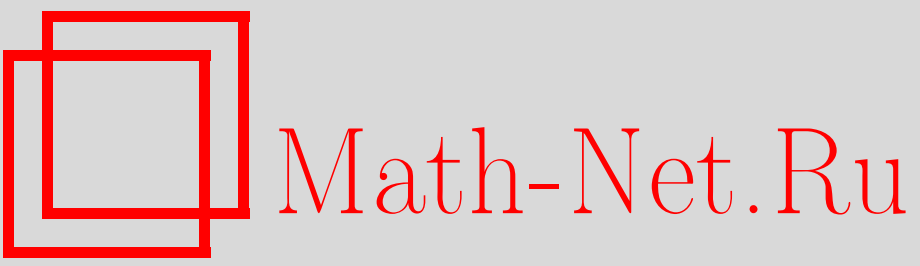

П. В. Гапеев, Задачи последовательного различения гипотез для составного пуассоновского процесса с экспоненциальными скачками, УМН, 2002, том 57, выпуск 6, 171-172

DOI: https://doi.org/10.4213/rm577

Использование Общероссийского математического портала Math-Net.Ru подразумевает, что вы прочитали и согласны с пользовательским соглашением

http://www.mathnet.ru/rus/agreement

Параметры загрузки:

IP: 18.208 .226 .222

26 апреля 2023 г., 18:34:49 


\title{
ЗАДАЧИ ПОСЛЕДОВАТЕЛЬНОГО РАЗЛИЧЕНИЯ ГИПОТЕЗ ДЛЯ СОСТАВНОГО ПУАССОНОВСКОГО ПРОЦЕССА С ЭКСПОНЕНЦИАЛЬНЫМИ СКАЧКАМИ
}

\author{
П. В. Г АПЕЕВ
}

Задача последовательного различения двух простых гипотез о распределении наблюдаемого процесса состоит в том, чтобы последовательно и с наименьшими потерями определить, какая из гипотез на самом деле выполнена. Постановка данной задачи для случая наблюдаемой последовательности независимых случайных величин была дана в [1]. С помощью байесовского подхода в статьях [2], [3] доказьвалась оптимальность критерия отношения вероятностей в вариационной постановке задачи для наблюдаемой последователњности величин. В работах [4; гл. IV] и [5] были даны точные решения байесовских и вариационных задач последовательного различения гипотез о среднем значении наблюдаемого винеровского процесса и об интенсивности наблюдаемого пуассоновского процесса соответственно. В данной работе дается точное решение байесовской задачи и явный вид границ критерия отношения правдоподобия в вариационной задаче последовательного различения гипотез для составного пуассоновского процесса с экспоненциальными скачками. Также даются точные формулы для вероятностей ошибок первого и второго рода и средней продолжителњности наблюдений для указанного критерия.

1. Предположим, что имеется некоторый стохастический базис $\left(\Omega, \mathscr{F},\left(\mathscr{F}_{t}\right)_{t \geqslant 0}, \mathrm{P}_{0}, \mathrm{P}_{1}\right)$ и доступньй наблюдению непрерьвно поступающий процесс $X=\left(X_{t}\right)_{t \geqslant 0}, X_{t}=\sum_{j=1}^{N_{t}} \xi_{j}, t>0$, $X_{0}=0$, где относительно меры $\mathrm{P}_{i}, i=0,1, N=\left(N_{t}\right)_{t \geqslant 0}$ - пуассоновский процесс интенсивности $1 / \lambda_{i}, \lambda_{i}>0$, и $\left(\xi_{j}\right)_{j \in \mathbb{N}}$ - последовательность независимых случайных величин, экспоненциально распределенных с параметром $\lambda_{i}\left(N\right.$ и $\left(\xi_{j}\right)_{j \in \mathbb{N}}$ предполагаются взаимно независимњми относительно $\mathrm{P}_{i}$ ). Процесс $X$ будем называть составным пуассоновским процессом с әкспоненциальными скачками (с параметром $\left.\lambda_{i}>0, i=0,1\right)$.

Для постановки байесовской задачи предположим, что на данном стохастическом базисе задана $\mathscr{F}_{0}$-измеримая случайная величина $\lambda \in\left\{\lambda_{0}, \lambda_{1}\right\}$, имеющая относительно меры $\mathrm{P}_{\pi}=\pi \mathrm{P}_{1}+$ $(1-\pi) \mathrm{P}_{0}$ распределение $\mathrm{P}_{\pi}\left[\lambda=\lambda_{1}\right]=\pi, \mathrm{P}_{\pi}\left[\lambda=\lambda_{0}\right]=1-\pi, \pi \in[0,1]$. Задача состоит в том, чтобы последовательно и с наименьшими потерями различить гипотезы $H_{1}: \lambda=\lambda_{1}$ и $H_{0}$ : $\lambda=\lambda_{0}$ (без ограничения общности $\lambda_{0}>\lambda_{1}>0$ ). Для этого рассмотрим решающее правило $(\tau, d)$, состоящее из момента остановки $\tau=\tau(\omega)$ относительно фильтрации $\left(\mathscr{F}_{t}^{X}\right)_{t \geqslant 0}$, где $\mathscr{F}_{t}^{X}=\sigma\left\{X_{s}: 0 \leqslant s \leqslant t\right\}, t \geqslant 0$, и $\mathscr{F}_{\tau}^{X}$-измеримой функции $d=d(\omega) \in\{0,1\}$. Будем полагать, что при остановке наблюдений в момент $\tau$ функция терминалњного решения показьвает, какую из гипотез $-H_{1}$ или $H_{0}$ - следует принять, согласно правилу: если $d=1$, принимаем $H_{1}$, а если $d=0$, то принимаем $H_{0}$.

Байесовская задача последовательного различения гипотез состоит в нахождении функции риска $V(\pi)=\inf _{(\tau, d)}\left\{\mathrm{E}_{\pi}[\tau]+a \pi \mathrm{P}_{1}[d=0]+b(1-\pi) \mathrm{P}_{0}[d=1]\right\}$, минимизирующей общие средние потери, связанные с решающим правилом $(\tau, d)$, состоящие из средней продолжительности наблюдений $\mathrm{E}_{\pi}[\tau]$ и потерь, связанных с неправильньм выбором заключительного решения, $a \pi \mathrm{P}_{1}[d=0]+b(1-\pi) \mathrm{P}_{0}[d=1]$, где $a, b>0$ - некоторые постоянные. Также требуется определить оптимальное ( $\pi$-байесовское) решающее правило $\left(\tau_{*}, d_{*}\right)$, при котором данный инфимум достигается.

Согласно рассуждениям в [4; гл. IV] данная задача сводится к задаче об оптималњной остановке $V(\pi)=\inf _{\tau} \mathrm{E}_{\pi}\left[\tau+a \pi_{\tau} \wedge b\left(1-\pi_{\tau}\right)\right]$ для процесса апостериорной вероятности $\pi=\left(\pi_{t}\right)_{t \geqslant 0}, \pi_{t}=\mathrm{P}_{\pi}\left[\lambda=\lambda_{1} \mid \mathscr{F}_{t}^{X}\right], t \geqslant 0, \pi_{0}=\pi$, и оптимальная решающая функция имеет вид: $d_{*}=1$ при $\pi_{\tau_{*}} \geqslant b /(a+b)$ и $d_{*}=0$ при $\pi_{\tau_{*}}<b /(a+b)$.

Отметим, что процесс $\pi=\left(\pi_{t}\right)_{t \geqslant 0}$ допускает представление $\pi_{t}=\pi e^{Z_{t}} /\left(1-\pi+\pi e^{Z_{t}}\right)$, где прочесс логарифма отношения правдоподобия $Z=\left(Z_{t}\right)_{t \geqslant 0}, Z_{t}=\ln \left[d\left(\mathrm{P}_{1} \mid \mathscr{F}_{t}^{X}\right) /\right.$ $\left.d\left(\mathrm{P}_{0} \mid \mathscr{F}_{t}^{X}\right)\right]$, в данном случае имеет вид $Z_{t}=\left(\lambda_{0}-\lambda_{1}\right) X_{t}-\left[\left(\lambda_{0}-\lambda_{1}\right) /\left(\lambda_{0} \lambda_{1}\right)\right] t, t \geqslant 0$.

Теорема 1. Предположим, что $1 / \lambda_{1}-1 / \lambda_{0}>1 / a+1 / b$. Тогда в данной байесовской задаче функция риска $V(\pi)=f\left(\pi ; B_{*}\right)$ при $\pi \in\left(A_{*}, B_{*}\right)$ u $V(\pi)=a \pi \wedge b(1-\pi)$ 
nри $\pi \in\left[0, A_{*}\right] \cup\left[B_{*}, 1\right]$, zде $f\left(\pi ; B_{*}\right)=b(1-\pi)+\lambda_{0} \lambda_{1}\left[\lambda_{1}\left(1-B_{*}\right)-\lambda_{0} B_{*}\right]\left(B_{*}-\pi\right) /$ $\left[\left(\lambda_{0}-\lambda_{1}\right)^{2} B_{*}\left(1-B_{*}\right)\right]+\lambda_{0} \lambda_{1}\left[\lambda_{0}(1-\pi)-\lambda_{1} \pi\right] \ln \left[\pi\left(1-B_{*}\right) /\left((1-\pi) B_{*}\right)\right] /\left(\lambda_{0}-\lambda_{1}\right)^{2}$, onтимальное решающее правило $\left(\tau_{*}, d_{*}\right)$ имеет вид $\tau_{*}=\inf \left\{t \geqslant 0: \pi_{t} \notin\left(A_{*}, B_{*}\right)\right\}, d_{*}=1$, если $\pi_{\tau_{*}} \geqslant B_{*}, d_{*}=0$, если $\pi_{\tau_{*}}=A_{*}$, и границьь $A_{*}$ и $B_{*}, 0<A_{*}<b /(a+b)<B_{*}<1$, однозначно определяются из системы трансцендентных уравнений $f\left(A_{*} ; B_{*}\right)=a A_{*} u$ $f_{\pi}^{\prime}\left(A_{*} ; B_{*}\right)=a$.

Заметим, что в случае $1 / \lambda_{1}-1 / \lambda_{0} \leqslant 1 / a+1 / b$ оптимальное $\pi$-байесовское решающее правило $\left(\tau_{*}, d_{*}\right)$ тривиально, т.е. $\tau_{*} \equiv 0, d_{*}=1$, если $\pi_{\tau_{*}} \geqslant b /(a+b)$, и $d_{*}=0$, если $\pi_{\tau_{*}}<b /(a+b)$.

2. Для постановки соответствующей вариационной задачи будем предполагать, что заданы числа $\alpha, \beta>0, \alpha+\beta<1$, и рассмотрим класс решающих правил $\Delta(\alpha, \beta)=\{(\tau, d)$ : $\left.\mathrm{P}_{1}[d=0] \leqslant \alpha, \mathrm{P}_{0}[d=1] \leqslant \beta\right\}$.

Вариационная задача последовательного различения гипотез состоит в нахождении в классе $\Delta(\alpha, \beta)$ решающего правила $(\widehat{\tau}, \widehat{d})$ такого, что $\mathrm{E}_{i}[\widehat{\tau}] \leqslant \mathrm{E}_{i}[\tau], i=0,1$, одновременно для всех $(\tau, d) \in \Delta(\alpha, \beta)$. Отметим, что в данной постановке не делается никаких предположений о распределении случайной величины $\lambda$.

Рассмотрим решающее правило $(\widehat{\tau}, \widehat{d})$, в котором $\widehat{\tau}=\inf \left\{t \geqslant 0: Z_{t} \notin(A, B)\right\}, \widehat{d}=1$, если $Z_{\widehat{\tau}} \geqslant B$, и $\widehat{d}=0$, если $Z_{\widehat{\tau}}=A$, с некоторьми $A$ и $B, A<0<B$. Такого рода решающее правило $(\widehat{\tau}, \widehat{d})$ назьвают критерием отношения правдоподобия. Оптимальность критерия отношения правдоподобия для случая, когда процесс $Z=\left(Z_{t}\right)_{t \geqslant 0}$ имеет стационарные независимые приращения, была доказана в работе [6].

Пару границ $(\alpha, \beta)$ будем называть допустимой, если для них найдутся такие $A$ и $B$, $A<0<B$, что вероятности ошибок первого и второго рода $\mathrm{P}_{1}[\widehat{d}=0]=\alpha$ и $\mathrm{P}_{0}[\widehat{d}=1]=\beta$. Множество всех допустимых пар $(\alpha, \beta)$ в соответствующей вариационной задаче для пуассоновского процесса было описано в статье [5].

Теорема 2. Предположим, что заданные $\alpha$ и $\beta$ принадлежат множеству допустимых пар $\mathscr{A}=\left\{(\alpha, \beta): 0<\alpha<1,0<\beta<\lambda_{1}(1-\alpha) / \lambda_{0}\right\}$. Тогда определенный выше критерий отношения правдоподобия $(\widehat{\tau}, \widehat{d})$ с $A=\ln [\alpha /(1-\beta)]$ и $B=\ln \left(\lambda_{1} / \lambda_{0}\right)+\ln [(1-\alpha) / \beta]$ является оптимальным в данной вариачионной задаче.

Заметим, что если $(\alpha, \beta) \notin \mathscr{A}$, то решающее правило $(\widehat{\tau}, \widehat{d})$ не является оптимальным в данной вариационной задаче.

Теорема 3. В условиях теоремы 2 для решающего правила $(\widehat{\tau}, \widehat{d})$ имеют место выражения $\mathrm{P}_{1}[\widehat{d}=0]=e^{A}\left(\lambda_{0} e^{B}-\lambda_{1}\right) /\left(\lambda_{0} e^{B}-\lambda_{1} e^{A}\right), \mathrm{P}_{0}[\widehat{d}=1]=\lambda_{1}\left(1-e^{A}\right) /\left(\lambda_{0} e^{B}-\lambda_{1} e^{A}\right)$ и средняя продолжительность наблюдений при гипотезах $H_{0}$ и $H_{1}$ имеет вид $\mathrm{E}_{0}[\widehat{\tau}]=$ $\lambda_{0} \lambda_{1}\left[\lambda_{1}\left(1-e^{A}\right)\left(\lambda_{1}-\lambda_{0}(1+B)\right)-\lambda_{0} A\left(\lambda_{0} e^{B}-\lambda_{1}\right)\right] /\left[\left(\lambda_{0}-\lambda_{1}\right)^{2}\left(\lambda_{0} e^{B}-\lambda_{1} e^{A}\right)\right] u \mathrm{E}_{1}[\widehat{\tau}]=$ $\lambda_{0} \lambda_{1}\left[\lambda_{0} e^{B}\left(\lambda_{0}+\lambda_{1}(B-1)\right)-\lambda_{1}^{2} e^{A} A-\lambda_{0} e^{A+B}\left(\lambda_{0}+\lambda_{1}(B-A-1)\right)\right] /\left[\left(\lambda_{0}-\lambda_{1}\right)^{2}\left(\lambda_{0} e^{B}-\lambda_{1} e^{A}\right)\right]$ соответственно.

Доказательства вышеуказанных утверждений содержатся в диссертации [7].

\section{СПИСОК ЛИТЕРАТУРЫ}

[1] А. Вальд. Последовательный анализ. М.: Физматгиз, 1960. [2] A. Wald, J. Wolfowitz // Ann. Math. Statist. 1948. V. 19. P. 326-339. [3] A. Wald, J. Wolfowitz // Ann. Math. Statist. 1950. V. 21. P. 82-99. [4] А. Н. Ширяев. Статистический последовательный анализ. M.: Наука, 1976. [5] G. Peskir, A. N. Shiryaev // Ann. Statist. 2000. V. 28. P. 837-859. [6] A. Irle, N. Schmitz // Math. Operationsforsch. Statist. Ser. Statist. 1984. V. 15. № 1. P. 91-104. [7] П. В. Гапеев // Дисс. ... канд. физ.-матем. наук. М.: МГУ, 2001. 